\title{
Bearing Fault Classification based on the Adaptive Orthogonal Transform Method
}

\author{
Mohamed Azergui, Abdenbi Abenaou and Hassane Bouzahir \\ Laboratory of Systems Engineering and Information Technology (LISTI) \\ National School of Applied Science, Ibn Zohr University, PO Box 1136, 80000 Agadir, Morocco
}

\begin{abstract}
In this work, we propose an approach based on building an adaptive base which permits to make accurate decisions for diagnosis. The orthogonal adaptive transformation consists of calculating the adaptive operator and the standard spectrum for every state, using two sets of vibration signal records for each type of fault. To classify a new signal, we calculate the spectral vector of this signal in each base. Then, the similarity between this vector and other standard spectra is computed. The experimental results show that the proposed method is very useful for improving the fault detection.
\end{abstract}

Keywords-Condition monitoring; vibration analysis; adaptive orthogonal transformation; bearing fault

\section{INTRODUCTION}

The rolling bearing is one of the most widely used elements in rotating machinery. As a critical component, it carries most of the load during the running of rotating machinery. If the rolling bearing fails, serious problems arise, which will, in turn, result in the decrease of production efficiency and large economic loss. Records show that faulty bearings contribute to about thirty percent of the failures in rotating machinery [1]. As a result, it is of great importance to study the effective fault diagnosis approaches for rolling bearings.

Various monitoring have been developed for bearing fault diagnosis and condition monitoring, such as vibration analysis, temperature and acoustic emission monitoring [2]. Vibration signal analysis is one of the most efficient techniques thanks to the useful information to severity and type of bearing damage [3], [4]. Various signal processing techniques have been proposed for mechanical fault diagnosis are time domain [5], frequency domain [6]-[8], time-frequency domain analysis [9], high frequency resonance technique (HFRT) [10], [11], wavelet transform methods [12], [13] and automatic diagnosis techniques [14]. In summary, such methods can be primarily categorized into two classes: frequency identification and features classification.

The basic idea of these methods is the decomposition of the vibration signal in a system of function of orthogonal base as those of Fourier, Walsh or Haar, [15]-[17] to obtain the vector (spectre) of the informative characteristics. However, the spectrum obtained by these frequency methods in the majority of cases will complicate the procedure of comparing the signals of various types of faults, since the vibration signal is a nonstationary process. Hence the need for a method of computing the vector of the informative characteristics with a minimum dimension.

In this paper, for the first time, we propose to use the adaptive orthogonal transformations for the extraction of the informative characteristics of bearing vibration signal. This method was used for voice signals [18] and was recently employed for classification of breast masses in mammography [19].

The use of these transformations is favored by the ability to adapt the shape of their basic functions according to the character of the standard vector. The latter is formed from the vibration signals of each fault type. In other words, each class of defects is associated with a system of basic functions adaptive for the projection of the signals. The formed basic function system is expressed as a factorization orthogonal matrix operator, which allows making a transformation with a fast calculation algorithm.

This paper is organized as follows. The principles of adaptive orthogonal transforms are introduced in Section II. The proposed method is validated using the data collected from bearing run-to-failure tests in Section III. Finally, the main conclusions are outlined in Section IV.

\section{THEORETICAL BACKGROUND}

In digital treatment, transformed shelf space orthogonal of a signal $X$ can be represented by the matrix (1).

$$
Y=\frac{1}{N} H X
$$

Where,

- $X=\left[x_{1}, x_{2}, \ldots \ldots \ldots, x_{N}\right]^{T}$ is the initial signal is to be transformed (of size $N=2^{n}$ ).

- $Y=\left[y_{1}, y_{2}, \ldots \ldots \ldots, y_{N}\right]^{T}$ is the vector of the spectral coefficients calculated by the operator orthogonal $\mathrm{H}$ of dimension $N \times N$.

To avoid the problem of signals synchronizations, we mention that $X$ is transformed to the Frequency domain.

Factorization of Good [20] showed a possibility of representing the matrix operator $\mathrm{H}$ as product $G_{i}$ (2) sparse matrix with a higher proportion of zero which has allowed the construction of the quick transformation algorithms of Fourier, Haar, and Walsh. The matrices $G_{i}(i=1, \ldots, n)$ are constructed by blocks of matrices $V_{i, j}$ of minimum dimension that is called spectral nuclei: 


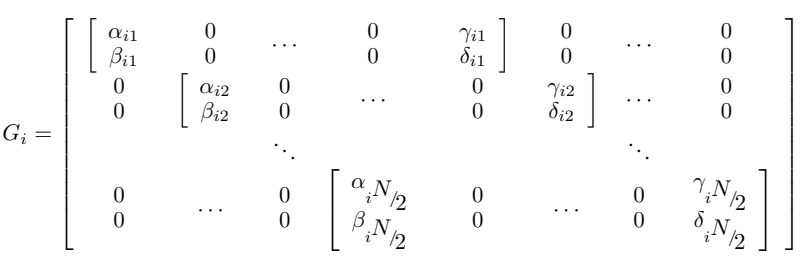

With

$$
\begin{gathered}
v_{i, j}=\left[\begin{array}{ccc}
\alpha_{i j} & \ldots & \gamma_{i j} \\
\beta_{i j} & \ldots & \delta_{i j}
\end{array}\right]=\left[\begin{array}{ccc}
\cos \left(\alpha_{i j}\right) & \ldots & w_{i, j} \sin \left(\alpha_{i j}\right) \\
\sin \left(\alpha_{i j}\right) & \ldots & -w_{i, j} \cos \left(\alpha_{i j}\right)
\end{array}\right], \\
w_{i, j}=\exp \left(j \theta_{i, j}\right), \varphi \in[0,2 \pi], \theta \in[0,2 \pi]
\end{gathered}
$$

Hence $((1))$ can be written as follows:

$$
Y=\frac{1}{N} H X=\frac{1}{N} G_{1} G_{2} \ldots G_{n} X=\frac{1}{N} \prod_{i=1}^{n} G_{i} X
$$

By defining the angular parameters, $\varphi_{i, j}$ and $\theta_{i, j}$, the operators of orthogonal transformations $\mathrm{H}$ can be formed with basic functions complex, or with real functions when $\theta_{i, j}=0$. The calculation of the parameters depends $\varphi_{i, j}$ on the choice of the structures of the spectral nuclei $V_{i, j}$. What allows generating a system of basic functions adapted to a given class of signals.

Yet, to assure a fast calculation, in this work, the spectral nuclei in matrices $G_{i}$ are established so that they contain a higher proportion of zeros, such as he is explained below.

Adapting operator $\mathrm{H}$ in (1) is provided by the condition:

$$
\frac{1}{N} H_{a} Z_{c d}=Y_{c}=\left[y_{c, 1}, 0,0, \ldots 0\right]^{T}, y_{c, 1} \neq 0
$$

Where,

- $Y_{c}$ is the target vector which builds the criterion of adaptation of the operator $H_{a}$.

- $\quad Z_{c d}$ represents the vector standard of a class calculated by means of the statistical characteristics of several vibratory signals.

- $H_{a}$ is adaptable to synthesize operator.

The synthesis of the adaptable operator Ha based standard $Z_{c d}$ (for a given class), consists in calculating the angular parameters $\varphi_{i, j}$ matrices $G_{i}$ according to the condition (4). The procedure of the calculation of the parameters is illustrated by Fig. 1 the principle of which is based on an iterative algorithm introduced by Fig. 2, which allows the calculation of the target vector $Y_{c}$ is according to the equation:

$$
Y_{i}=G_{i} Y_{i-1}
$$

The calculation of the vector $Y_{c}$ allows the obtaining of the adapted operator $\mathrm{H}$. For the classification of the vibration signals, we dispose two sets of the vibration signals. The first

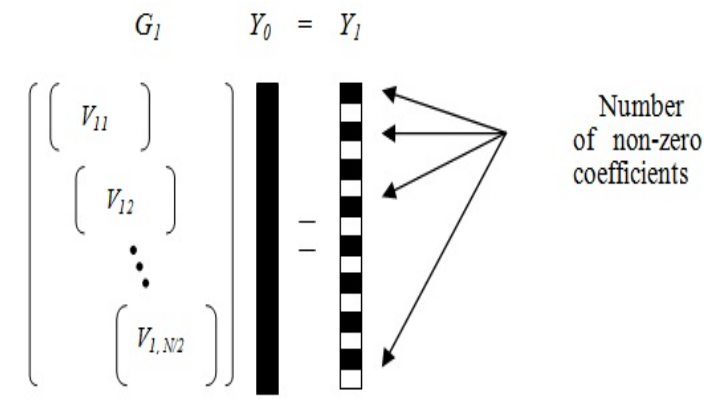

The second iteration

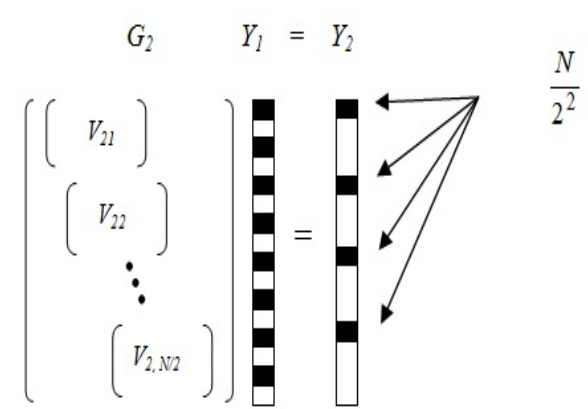

Thenth

iteration
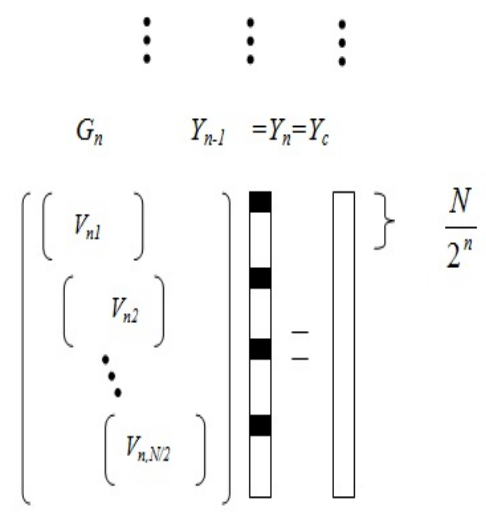

$H_{a}=G_{n} G_{n-l} \ldots G_{l}$

Fig. 1. The procedure of synthesis of the operator of the adaptive transformed

one serves to calculate the standard $Z_{c d}$ of $\mathrm{i}$ (class i) and allows to generate the synthesis of the operator. Whereas the second set used to form the spectral standard $Y_{s d, i}$ of $\mathrm{i}$, which is obtained by the projection of the recordings of the second set in the adaptable base $H_{a}$.

To make the decision and classify vibration signal, we calculate each $Y_{i}$ spectrum in each base $H_{a, i}$. To define the fault corresponding to the vector $\mathrm{Yi}$ of the informative characteristics, we lean on a rule of decision formed by a combination of two criteria:

- The Euclidean distance $\delta_{i}=\left\|Y_{i}-Y_{s d, i}\right\|$ and

- The distance of the energy concentrated in their first coefficients of the decomposition $\varepsilon_{i}=\left|Y_{1, i}^{2}-Y_{1, s d, i}^{2}\right|$.

So, the vector $Y_{i}$ will correspond to class $i$ if $\delta_{i}=$ $\min \left(\delta_{k=1 \ldots M}\right)$ and $\varepsilon_{i}=\min \left(\varepsilon_{k=1 \ldots M}\right)$, with $M$ is the number of classes. This procedure of classification is illustrated 


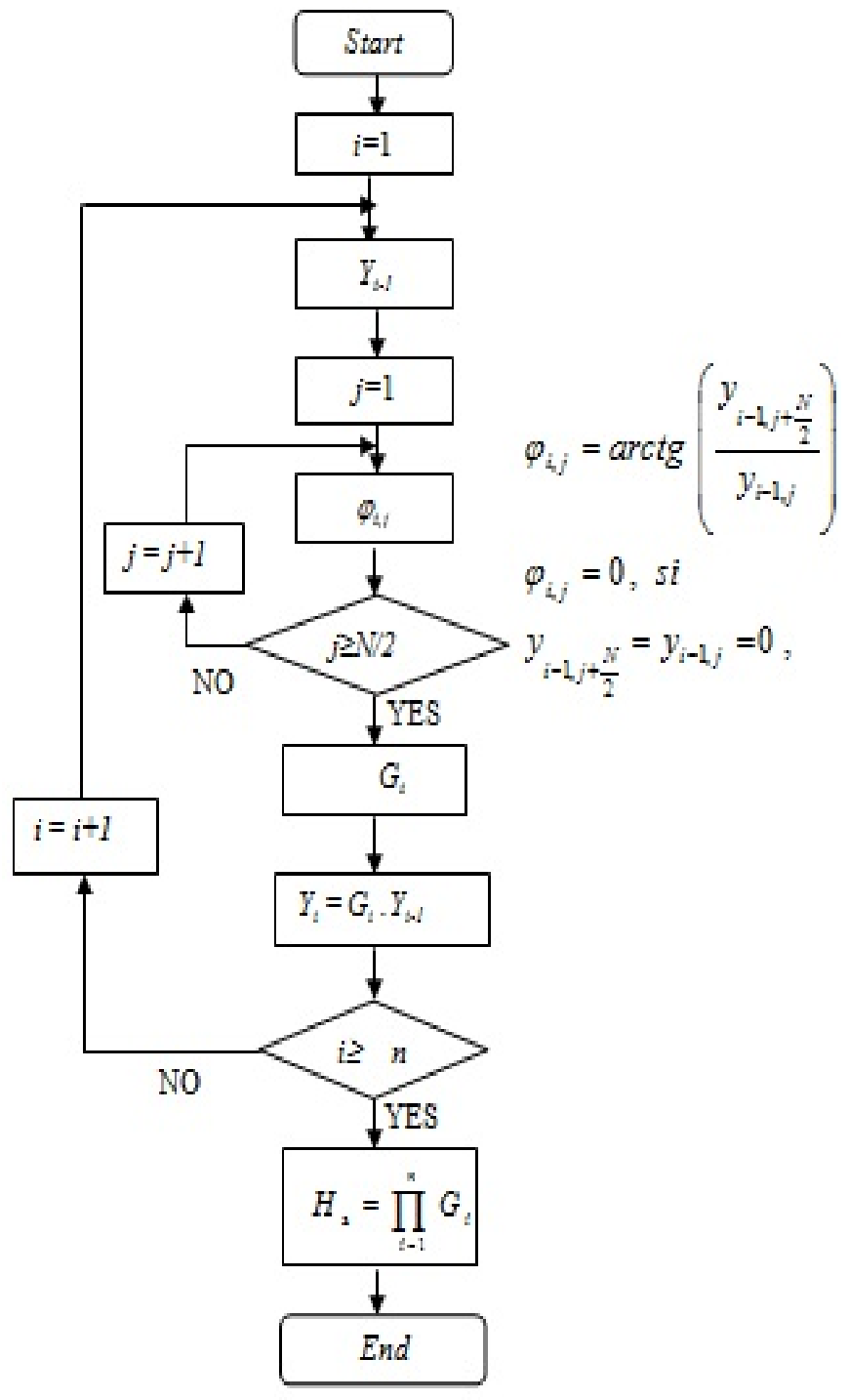

Fig. 2. The algorithm of synthesis of the operator of the adaptive transformed.

in the Fig. 3.

\section{APPlicAtion to EXPERIMENTAL SignAL}

\section{A. Experimental setup}

The bearing test rig hosts four bearings were installed on a shaft. The rotation speed was kept constant at a rate of 2000 RPM by an alternative current motor coupled to the shaft via rub belts. A uniform radial load of $6000 \mathrm{lbs}$ is applied onto the shaft and bearing. All bearings are lubricated.

Rexnord ZA-2115 double row bearings were installed on the shaft as shown in Fig. 4. A PCB 353B33 High Sensitivity Quartz ICP accelerometers were installed on the bearing housing. The test rig and sensors placement are also shown in Fig. 4. All failures occurred after exceeding designed lifetime of the bearing which is more than 100 million revolutions. Vibration data were collected every 10 minutes by NI DAQCard$6062 \mathrm{E}$ at the sample rate set at $20 \mathrm{KHz}$.

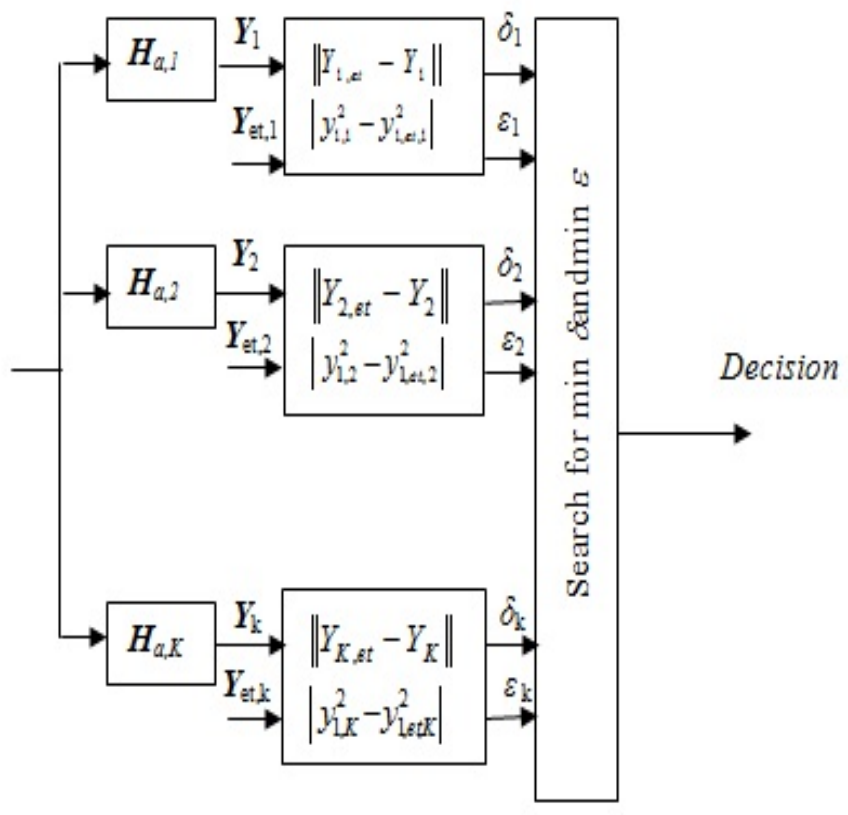

Fig. 3. Classification procedure.

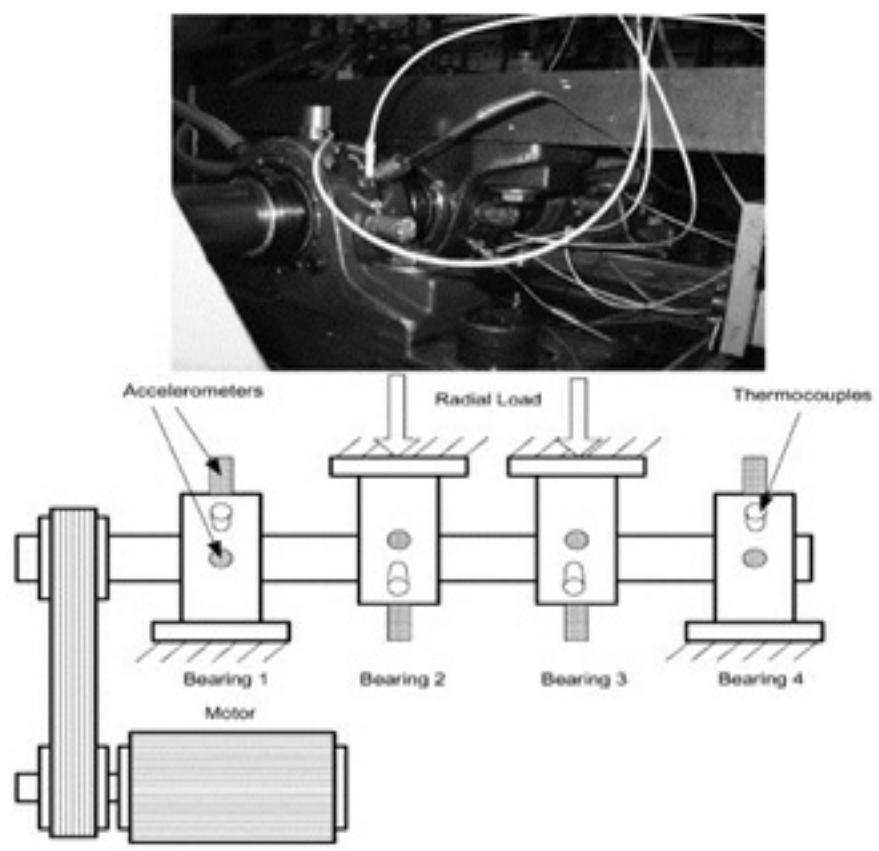

Fig. 4. Bearing test rig.

The test was carried out for 35 days until a significant amount of metal debris was found on the magnetic plug of the test bearing. An inner race defect was discovered in test bearing 1 .

\section{B. Experimental Results Analysis}

The proposed method was applied to detect the bearing with outer race fault. The raw vibration signal of normal operating conditions and outer race failure occurred in bearing 1 are plotted in 


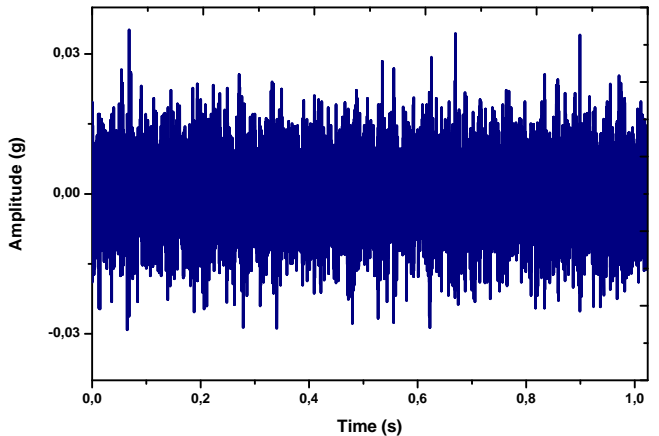

(a)

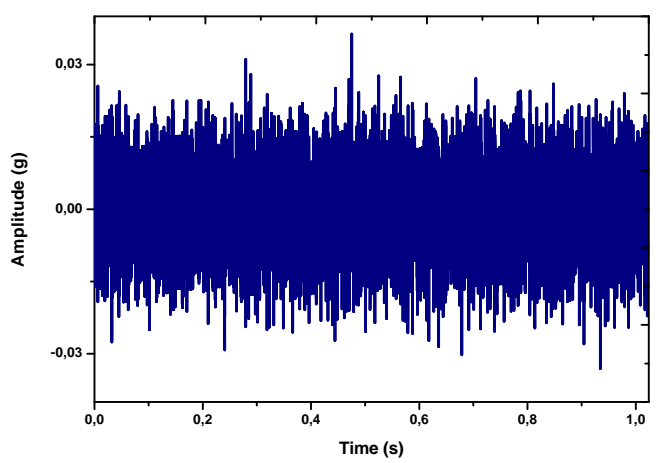

(b)

Fig. 5. Vibration signal of: (a) normal operating conditions, (b) outer race failure occurred in bearing 1 .

Fig. 5a and 5b, respectively.

Fig. $6 \mathrm{a}$ and $6 \mathrm{~b}$ present the frequency spectrum of normal state and outer race. The characteristic defect frequencies cannot be obtained directly in FFT spectrum.

By using the elaborate method, the projection of normal signal in the normal class base and fault class base are plotted in Fig. 7a and 7b, respectively. We can notice that the energy of the projection of the normal signal in the adaptive base has a small spectral vector (Fig. 7a).

Fig. $8 \mathrm{a}$ and $8 \mathrm{~b}$ illustrate the projection of fault signal in the normal class base and fault class base. It can be seen that during the projection of this signal at a normal base, we obtain rather a broad spectral vector (Fig. 8a).

This result demonstrate that the first signal belongs to the class of the normal signal and the second signal belongs to the class of abnormal signal, respectively. The same conclusion also manifested by values of $\delta$ and $\varepsilon$.

The results obtained by the developed method, illustrated in Fig. 7 and 8 indicate its effectiveness and show that it ensures a high distinction that will help to make the classification of bearing vibration signal.

The efficiency of the elaborate method is illustrated on Fig. 9 which reflects the certainty of classification according to the size of the interval of the analysis. The certainty of the

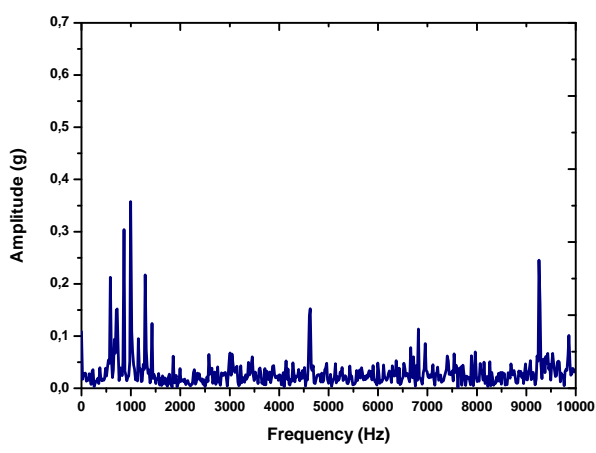

(a)

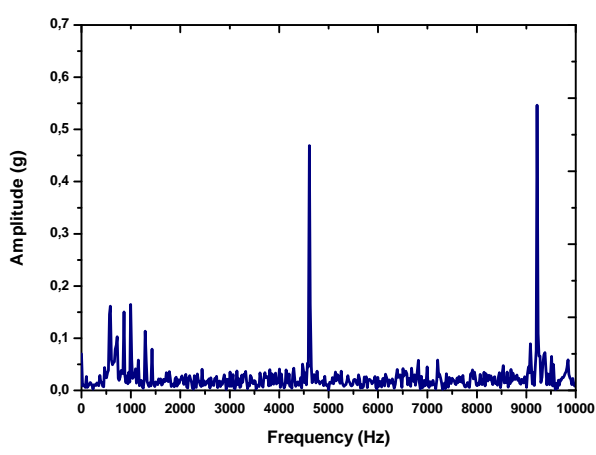

(b)

Fig. 6. FFT of: (a) normal operating conditions, (b) outer race failure occurred in bearing 1 .

classification of the signals is much higher and can reach a $100 \%$ value as the interval of analysis increases.

\section{CONCLUSION}

To improve the accuracy fault classification of bearings in rotating machines, a new method is developed based to calculate the informative characteristics of the vibration signal. The experimental results show that the method ensures a high distinction that will help to make the classification of bearing vibration signal. The developed software system according to this method will be beneficial for practical fault classification.

\section{REFERENCES}

[1] N. Tandon and A. Parey, "Condition monitoring of rotary machines," in Condition Monitoring and Control for Intelligent Manufacturing. Springer, 2006, pp. 109-136.

[2] W. Zhou, T. G. Habetler, and R. G. Harley, "Bearing condition monitoring methods for electric machines: A general review," in Diagnostics for Electric Machines, Power Electronics and Drives, 2007. SDEMPED 2007. IEEE International Symposium on. IEEE, 2007, pp. 3-6.

[3] N. Tandon and B. Nakra, "Comparison of vibration and acoustic measurement techniques for the condition monitoring of rolling element bearings," Tribology International, vol. 25, no. 3, pp. 205-212, 1992.

[4] N. Tandon and A. Choudhury, "A review of vibration and acoustic measurement methods for the detection of defects in rolling element bearings," Tribology international, vol. 32, no. 8, pp. 469-480, 1999.

[5] H. A. Khwaja, S. Gupta, and V. Kumar, "A statistical approach for fault diagnosis in electrical machines," IETE Journal of Research, vol. 56, no. 3, pp. 146-155, 2010. 


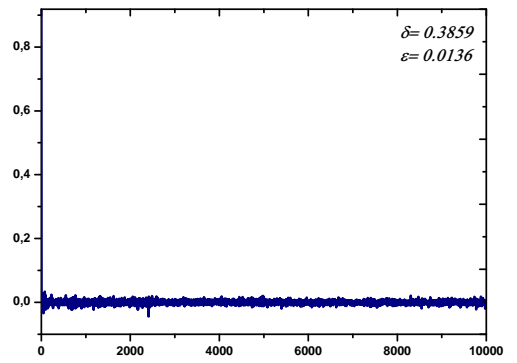

(a)

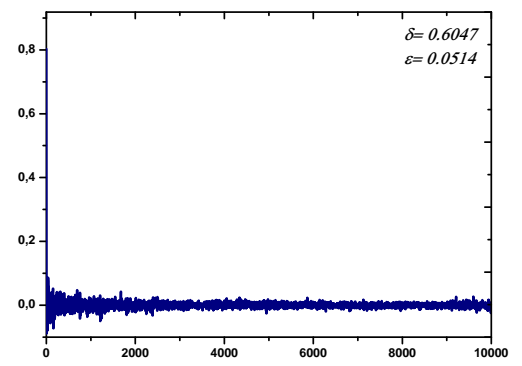

(b)

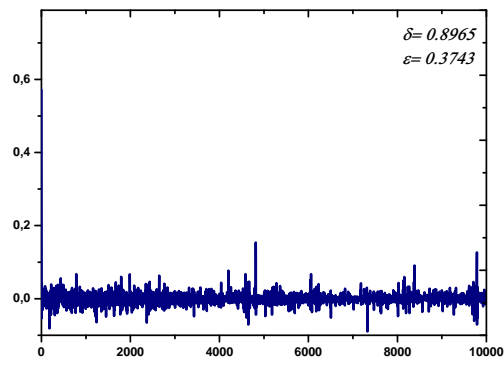

(a)

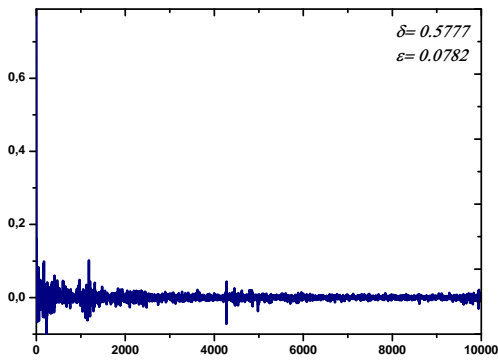

(b)

Fig. 7. Projection of normal signal in the: (a) normal class base, Fig. 8. Projection of fault signal in the: (a) normal class base,

(b) fault class base.

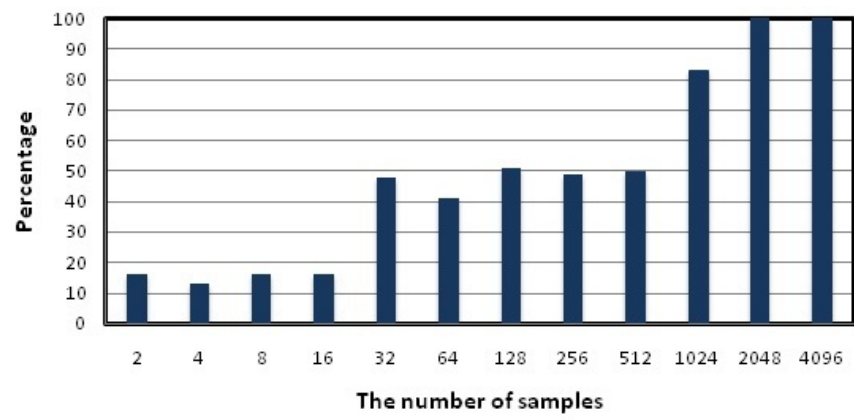

Fig. 9. Classification percentage according to the size of the interval of the analysis.

[6] E. Jantunen, "A summary of methods applied to tool condition monitoring in drilling," International Journal of Machine Tools and Manufacture, vol. 42, no. 9, pp. 997-1010, 2002.

[7] F. P. G. Márquez, A. M. Tobias, J. M. P. Pérez, and M. Papaelias, "Condition monitoring of wind turbines: Techniques and methods," Renewable Energy, vol. 46, pp. 169-178, 2012.

[8] S. Gowid, R. Dixon, and S. Ghani, "A novel robust automated fft-based segmentation and features selection algorithm for acoustic emission condition based monitoring systems," Applied Acoustics, vol. 88, pp. 66-74, 2015.

[9] J.-H. Lee, J. Kim, and H.-J. Kim, "Development of enhanced wignerville distribution function," Mechanical systems and signal processing, vol. 15 , no. 2, pp. 367-398, 2001. (b) fault class base.

[10] P. McFadden and J. Smith, "Vibration monitoring of rolling element bearings by the high-frequency resonance techniquea review," Tribology international, vol. 17, no. 1, pp. 3-10, 1984.

[11] T.-C. Liu and T.-Y. Wu, "Application of empirical mode decomposition and envelop analysis to fault diagnosis in roller bearing with single/double defect," Smart Science, vol. 5, no. 3, pp. 150-159, 2017.

[12] N. Nikolaou and I. Antoniadis, "Rolling element bearing fault diagnosis using wavelet packets," Ndt \& E International, vol. 35, no. 3, pp. 197205, 2002.

[13] Y. Jiang, B. Tang, Y. Qin, and W. Liu, "Feature extraction method of wind turbine based on adaptive morlet wavelet and svd," Renewable energy, vol. 36, no. 8, pp. 2146-2153, 2011.

[14] P. Jayaswal and A. Wadhwani, "Application of artificial neural networks, fuzzy logic and wavelet transform in fault diagnosis via vibration signal analysis: A review," Australian Journal of Mechanical Engineering, vol. 7, no. 2, pp. 157-171, 2009.

[15] K. Rao and N. Ahmed, "Orthogonal transforms for digital signal processing," in Acoustics, Speech, and Signal Processing, IEEE International Conference on ICASSP'76., vol. 1. IEEE, 1976, pp. 136-140.

[16] H. Kekre, T. K. Sarode, P. Natu, and S. Natu, "Transform based face recognition with partial and full feature vector using dct and walsh transform," in Proceedings of the International Conference \& Workshop on Emerging Trends in Technology. ACM, 2011, pp. 1295-1300.

[17] N. Ahmed and K. R. Rao, Orthogonal transforms for digital signal processing. Springer Science \& Business Media, 2012.

[18] A. Abdenbi, A. A. Fadoua, and N. Benayad, "Vers un système de reconnaissance automatique de la parole en amazighe basé sur les transformations orthogonales paramétrables."

[19] K. El Fahssi, A. Elmoufidi, A. Abenaou, S. Jai-Andaloussi, and A. Sekkaki, "Feature extraction of the lesion in mammogram images using segmentation by minimizing the energy and orthogonal transformation adaptive," WSEAS TRANSACTIONS on BIOLOGY and BIOMEDICINE, vol. 11, 2014.

[20] I. J. Good, "The interaction algorithm and practical fourier analysis," 
(IJACSA) International Journal of Advanced Computer Science and Applications, Vol. 9, No. 1, 2018

Journal of the Royal Statistical Society. Series B (Methodological), pp.

361-372, 1958. 